\title{
Pacific
}

Journal of

Mathematics

\section{NILPOTENT CHARACTERS}

GABRIEL NAVARRO

Volume $169 \quad$ No. 2

June 1995 


\title{
NILPOTENT CHARACTERS
}

\author{
GABRIEL NAVARRO
}

In this note we study modular characters of finite $p$ solvable groups which are induced from $p$-nilpotent subgroups and its $\pi$-version.

1. Introduction. There is at least one reason to study such characters. In [2], for any block $B$ of a finite group $G$, Alperin and Broué found a successful and natural Sylow $B$-theory which synthesized local group theory with several results on blocks by Brauer. This approach led to the Broué-Puig idea of nilpotent blocks. From the local representation point of view, therefore, the nilpotent blocks are the most natural blocks.

It is well known that theorems on $p$-blocks, in general, become far more accessible when we restrict our attention to the $p$-solvable groups. Sometimes, as it happens with the cyclic defect theory, they almost become trivial. This is not the case with the nilpotent blocks. Puig described the block algebra of a nilpotent block of a $p$-solvable group in [11].

Here we focus ourselves with the characters of the block. If $\varphi$ is a modular character lying in a $p$-block $B$ of a finite $p$-solvable group, we show that $B$ is nilpotent if and only if $\varphi$ is induced from a $p$-nilpotent subgroup. With this approach and applying Isaacs $\pi$-theory we are able to introduce nilpotent $\pi$-blocks ( $\pi$-blocks have been studied by Robinson, Staszewski, Slattery and others) and to describe them satisfactorily: they only have a unique modular character $\varphi$ (which is induced from a subgroup $K$ with a normal Hall $\pi$-subgroup), and its $|\operatorname{Irr}(D)|$ ordinary characters are also induced from convenient characters of $K$ ( $D$ is defect group of the block). Finally, we will find the Fong characters associated with $\varphi$ (the characters $\alpha$ of a Hall $\pi$-subgroup with $\alpha^{G}=\Phi_{\varphi}$ ).

Of course, when the set of primes $\pi$ is just the complement of a prime $p, \pi$-blocks are just the ordinary blocks. 
2. Subpairs and nilpotent blocks. If $B$ is a $p$-block of a finite group $G$, a $B$-subpair is a pair $\left(P, b_{P}\right)$ where $P$ is a $p$-subgroup of $G$ and $b_{P}$ is a block of $P C_{G}(P)$ inducing $B$ (we are using Alperin's book notation [1] ). If $P$ is a defect group of $B$, then $\left(P, b_{P}\right)$ is said to be a Sylow $B$-subpair. It is one of the main results in [2] to show that Sylow $B$-subpairs are $G$-conjugate and that each $B$-subpair is contained in one Sylow $B$-subpair (a natural but not obvious definition of containment is given in [1]). It is worth to mention that if the block $B$ is the principal block, local block theory is just Sylow theory.

Inspired by Frobenius Theorem, Broue and Puig defined nilpotent blocks: a block $B$ is said to be nilpotent if whenever $\left(P, b_{P}\right)$ is a $B$ subpair then $N_{G}\left(P, b_{P}\right) / C_{G}(P)$ is a $p$-group.

We begin with a Lemma. It is not in general true that if $b^{G}$ is defined and nilpotent, then $b$ is nilpotent (we will give some example below). However, in some special conditions more is true. (We recall that notation used in [2] and [4], is entirely equivalent to that in [1]: just apply V. 3.5 of [5]).

LEMMA 1. Let $B$ be a block of a p-solvable group $G$. Let $\theta \in$ $\operatorname{Irr}\left(O_{p^{\prime}}(G)\right)$ be covered by $B$ and let $b \in B l(T)$ cover $\theta$ and induce $B$, where $T$ is the inertia group of $\theta$ in $G$. Then $B$ is nilpotent if and only if $b$ is nilpotent.

Proof. Suppose that $B$ is nilpotent and let $\left(P, b_{P}\right)$ be a $b$-subpair. We wish to show that $N_{T}\left(P, b_{P}\right) / C_{T}(P)$ is a $p$-group. Let us denote by $^{*}: \operatorname{Irr}_{P}(O) \rightarrow \operatorname{Irr}\left(C_{O}(P)\right)$ the Glauberman Correspondence (see Chapter 13 of [6]), where $O=O_{p^{\prime}}(G)$.

By applying, for instance, Lemma (4.4) of [13] to $T$, we have that if $b_{P}$ covers $\psi^{*}$, where $\psi \in \operatorname{Irr}_{P}(O)$, then $b$ covers $\psi$. Therefore, we have that $b_{P}$ lies over $\theta^{*}$. We observe that $N_{T}(P)$ is the inertia subgroup of $\theta^{*}$ in $N_{G}(P)$. This is because $N_{G}(P)$ acts on $O$ fixing the $P$-invariant characters and commuting with the correspondence (see Theorem (13.1) (c) of [6]).

By Theorem (1.2.4) of [4], we know that $b_{P}^{P C_{G}(P)}$ is nilpotent; so let $\delta$ be the unique Brauer character in $b_{P}^{P C_{G}(P)}$. Since $\delta$ lies over $\theta^{*}$ and $P C_{T}(P)$ is the inertia group of $\theta^{*}$ in $P C_{G}(P)$, let $\mu \in \operatorname{IBr}\left(P C_{T}(P) \mid \theta^{*}\right)$ such that $\mu^{P C_{G}(P)}=\delta$. By Fong-Reynolds (Theorem V.2.5 of [5]), we know that $\mu$ is the only modular char- 
acter in $b_{P}$. Therefore, if $x \in N_{T}\left(P, b_{P}\right)$ then $\mu^{x}=\mu, \delta^{x}=\delta$ and consequently $x \in N_{G}\left(P, b_{P}^{P C_{G}(P)}\right)$. Then $N_{T}\left(P, b_{P}\right) / C_{T}(P)$ is isomorphic to a subgroup of $N_{G}\left(P, b_{P}^{P C_{G}(P)}\right) / C_{G}(P)$, which is a $p$-group by hypothesis.

Now assume that $b$ is nilpotent and let $\left(P, b_{P}\right)$ be a $B$-subpair. We want to prove that $N_{G}\left(P, b_{P}\right) / C_{G}(P)$ is a $p$-group. Let $H=$ $P C_{G}(P)$. We note that $b_{P}^{H O}$ covers $\theta^{x}$, for some $x \in G$. This can be seen, for instance, by taking an irreducible character of $b_{P}^{H O}$ lying under some irreducible character of $B$ (by Theorem B of [3]). Since $P$ is contained in a defect group of $b_{P}^{H O}$, it follows that some $O$ conjugate of $P$, say $P^{o}$, stabilizes $\theta^{x}$, by Fong-Reynolds. Therefore, $P$ stabilizes $\theta_{1}$ and $b_{P}^{H O}$ covers $\theta_{1}$, where $\theta_{1}=\theta^{x o^{-1}}$. Let $T_{1}$ be the stabilizer of $\theta_{1}$ in $G$. If we denote by $\theta_{1}^{*} \in \operatorname{Irr}\left(C_{O}(P)\right)$ the Glauberman correspondent of $\theta_{1}$ with respect to $P$, by an earlier argument we have that $N_{T_{1}}(P)$ is the stabilizer of $\theta_{1}^{*}$ in $N_{G}(P)$.

Now let $\gamma^{*} \in \operatorname{Irr}\left(C_{O}(P)\right)$ be covered by $b_{P}$. Then $\gamma$ is covered by $b_{P}^{H O}$, and therefore $\gamma=\theta_{1}^{c}$, for some $c \in C_{G}(P)$. Thus $\theta_{1}^{*}=\left(\gamma^{*}\right)^{c^{-1}}$ is also covered by $b_{P}$. Since $P C_{T_{1}}(P)$ is the stabilizer in $P C_{G}(P)$ of $\theta_{1}^{*}$, we find $e \in B l\left(P C_{T_{1}}(P) \mid \theta^{*}\right)$ such that $e^{P C_{G}(P)}=b_{P}$. By an earlier argument, $e^{T_{1}}$ lies over $\theta_{1}$, and, since it induces $B$, it follows that $e^{T_{1}}$ is a $G$-conjugate of $b$. Therefore, it is nilpotent. By Theorem (1.2) of [4], $e$ is also nilpotent and thus it contains a unique modular character, say $\delta$. By Fong-Reynolds, $\delta^{P C_{G}(P)}$ is the unique modular character in $b_{P}$.

Suppose now that $y \in N_{G}\left(P, b_{P}\right)$. Then $y$ fixes $P$ and $\delta^{P C_{G}(P)}$. By Clifford Theory, $\left(\theta_{1}^{*}\right)^{y}=\left(\theta_{1}^{*}\right)^{c}$, for some $c \in C_{G}(P)$. Thus $y c^{-1} \in N_{T_{1}}(P)$ and by the uniqueness in the Clifford Correspondence, $\delta^{y c^{-1}}=\delta$. Then $y c^{-1} \in N_{T_{1}}(P, e)$. Consequently, $N_{G}\left(P, b_{P}\right) \subseteq$ $N_{T_{1}}(P, e) C_{G}(P)$. Thus $N_{G}\left(P, b_{P}\right) / C_{G}(P)$ is isomorphic to a subgroup of $N_{T_{1}}(P, e) / C_{T_{1}}(P)$, which is a $p$-group.

Lemma 2. Let $B$ be a nilpotent block of a p-solvable group $G$ and let $\theta \in \operatorname{Irr}\left(O_{p^{\prime}}(G)\right)$ covered by $B$. If $\theta$ is $G$-invariant then $G$ is p-nilpotent.

Proof. We argue by induction on $|G|$. Write $O=O_{p^{\prime}}(G)$.

By Fong Theory, (see, for instance, Theorem (2.1) of [13]), we know that the Sylow $p$-subgroups of $G$ are the defect groups of $B$. Fix $P$ a Sylow $p$-subgroup of $G$ and let $\left(P, b_{P}\right)$ be a Sylow $B$ - 
subpair. By Frobenius Theorem, it suffices to show that if $Q$ is any $p$-subgroup of $P$ then $N_{G}(P) / C_{G}(P)$ is a $p$-group. By Theorem $(16.3)$ of $[\mathbf{1}]$, let $\left(Q, b_{Q}\right) \leq\left(P, b_{P}\right)$. Since $b_{Q}$ is nilpotent, let $\delta$ be the unique Brauer character in $b_{Q}$. By earlier arguments in Lemma 1, if $\theta^{*} \in \operatorname{Irr}\left(C_{O}(Q)\right)$ is the $Q$-Glauberman correspondent of $\theta \in \operatorname{Irr}_{Q}(O)$, then $b_{Q}$ lies over $\theta^{*}$ and $\theta^{*}$ is $N_{G}(Q)$-invariant. By local group theory, it is well known that $C_{O}(Q)=O_{p^{\prime}}\left(N_{G}(Q)\right)$. If $Q C_{G}(Q)<G$, by induction, we have that $Q C_{G}(Q) / O_{p^{\prime}}\left(N_{G}(Q)\right)$ is a $p$-group. Therefore, by Green's Theorem (see, for instance, (3.1) of $[8]), \delta_{O_{p^{\prime}}\left(N_{G}(Q)\right)}=\theta^{*}$, and since $\delta$ is the only Brauer character lying over $\theta^{*}$, we have that $\delta$ and $\theta^{*}$ determine one each other uniquely. Therefore, $\delta$ is $N_{G}(Q)$-invariant, and so it is $b_{Q}$. Thus, $N\left(Q, b_{Q}\right) / C_{G}(Q)=N_{G}(Q) / C_{G}(Q)$ is a $p$-group in any case, and Frobenius Theorem applies.

3. $\pi$-characters. If $G$ is a $\pi$-separable group, we denote by $I_{\pi}(G)$ the set of Isaacs $\pi$-characters of $G$. Of course, when $\pi=$ $p^{\prime}, I_{\pi}(G)$ is just the set of Brauer characters of $G$. We refer the reader to [7] and [8], for definitions, notation and basic properties of the set $I_{\pi}(G)$. We recall that there exists a canonical subset of the irreducible characters of $G, B_{\pi}(G)$, such that restriction to $\pi$ elements gives a bijection from $B_{\pi}(G)$ onto $I_{\pi}(G)$ (Theorem (9.3) of $[7])$.

We certainly will use that any $\pi$-character is induced from a $\pi$ degree $\pi$-character (Huppert's Theorem, see (3.4) of [8]), and other fact proved recently in [9]. If $\varphi \in I_{\pi}(G)$ and $\varphi=\delta^{G}=\mu^{G}$, where $\delta \in I_{\pi}(K)$ and $\mu \in I_{\pi}(J)$ have $\pi$-degree, then the Hall $\pi^{\prime}$-subgroups of $K$ and $J$ are $G$-conjugate: this invariant is the vertex of a $\pi$ character.

We say that $\varphi \in I_{\pi}(G)$ is nilpotent if $\varphi=\delta^{G}$, where $\delta \in I_{\pi}(K)$ with $K=O_{\pi \pi^{\prime}}(K)$.

LEMmA 3. Let $G$ be a $\pi$-separable group and let $\varphi \in I_{\pi}(G)$ be nilpotent. If $\theta \in \operatorname{Irr}\left(O_{\pi}(G)\right)$ is $G$-invariant and lies under $\varphi$ then $G=O_{\pi \pi^{\prime}}(G)$.

Proof. Write $\varphi=\delta^{G}$, where $\delta \in I_{\pi}(K)$ with $K=O_{\pi \pi^{\prime}}(K)$, and let $O=O_{\pi}(G)$. Since $O K$ has a normal Hall $\pi$-subgroup, by replacing $(K, \delta)$ by $\left(O K, \delta^{O K}\right)$, we may assume that $O \subseteq K$. Now, by (3.4) 
of $[8]$, let $\beta \in I_{\pi}(R)$ with $\pi$-degree be such that $\beta^{K}=\delta$. Since $\beta^{O R}$ has also $\pi$-degree (because $|O R: R|$ is a $\pi$-number), we also may assume that $\delta$ has $\pi$-degree.

By comments above, observe that if $P$ is a Hall $\pi^{\prime}$-subgroup of $K$, then $P$ is a vertex of $\varphi$.

Let $U=O_{\pi \pi^{\prime}}(G)$. We claim that $\varphi_{U}=e \eta$, where $\eta \in I_{\pi}(U)$ and $\eta_{O}=\theta$. To see this, let $\chi \in B_{\pi}(G)$ be a lifting of $\varphi$ (see Theorem (9.3) of [7]), and let $\psi \in B_{\pi}(U)$ be under $\chi$ ((7.5) of [7]). Then, by (6.3) and (6.5) of [7], $\psi_{U}=\theta$ and $\psi$ is the only $B_{\pi}$-character lying over $\theta$. Therefore, $\psi$ is $G$-invariant and so it is $\psi^{o}=\eta \in I_{\pi}(U)$, its restriction to $\pi$-elements. This proves the claim.

Now, since $\psi$ has $\pi$-degree, by (5.4) of [7], $\psi$ is $\pi$-special and therefore, $(U, \psi)$ is a subnormal $\pi$-factorable pair in the sense of $[7]$. Therefore, $(U, \psi) \leq(W, \alpha)$, where $(W, \alpha), \alpha$ a $\pi$-special character of $W$, is a nucleous of $\chi$ (definition (4.6) of [7]). Thus $\alpha^{o^{G}}=\varphi$, and by Theorem $\mathrm{B}$ of $[\mathbf{9}]$, it follows that $P^{x}$ is a Hall $\pi^{\prime}$-subgroup of $W$, for some $x \in G$. Then $P^{x} \cap U$ is a Hall $\pi^{\prime}$-subgroup of $U$, and thus $U \subseteq O P \subseteq K$.

Now, since $U / O$ and $O_{\pi}(K) / O$ are normal subgroups of $K / O$ of coprime order it follows that $O_{\pi}(K) / O \subseteq C_{G / O}(U / O) \subseteq U / O$, by Lemma 1.2.3. Therefore, we conclude that $O_{\pi}(K)=O$. Let $V=O_{\pi \pi^{\prime} \pi}(G)$. Since $K / U$ and $V / U$ have coprime orders it follows that $V \cap K=U$. Observe that $\delta_{U}=\eta$, by (3.1) of [8], and that $\delta^{K V}$ has $\pi$-degree. Therefore, $\eta^{V}=\left(\delta^{K V}\right)_{V} \in I_{\pi}(V)$. Since $\psi$ lifts $\eta$, necessarily $\psi^{V} \in \operatorname{Irr}(V)$. Since $\psi$ is $G$-invariant, by problem (6.1) of [6], for instance, it follows that $U=V=G$, as wanted.

Lemma 4. Let $G$ be a $\pi$-separable group and let $Y$ be a normal $\pi$-subgroup of $G$. Let $\varphi \in I_{\pi}(G)$, let $\theta \in \operatorname{Irr}(Y)$ under $\varphi$ and let $\delta \in I_{\pi}(T \mid \theta)$ with $\delta^{G}=\varphi$, where $T$ is the stabilizer of $\theta$ in $G$. Then $\varphi$ is nilpotent if and only if $\delta$ is nilpotent.

Proof. By the definition, certainly $\varphi$ is nilpotent if $\delta$ is nilpotent. So assume that $\varphi$ is nilpotent and write $\varphi=\psi{ }^{G}$, where $\psi \in I_{\pi}(K)$, with $K$ having a normal Hall $\pi$-subgroup. Since $Y K$ has also -a normal Hall $\pi$-subgroup, we may replace $K$ by $Y K$ and assume that $K$ contains $Y$. Also, by replacing $K$ by some $G$-conjugate, we may assume that $\psi$ lies over $\theta$. If $\alpha \in I_{\pi}(K \cap T \mid \theta)$ induces $\psi$, by uniqueness in the Clifford correspondence, (3.2) of [8], it follows 
that $\alpha^{T}=\delta$, and the proof of the Lemma is complete.

Now we prove.

THEOREM 5. Let $B$ be a p-block of a p-solvable group and let $\varphi \in \operatorname{IBr}(B)$. Then $B$ is nilpotent if and only if $\varphi$ is nilpotent.

Proof. We argue by induction on $|G|$. Let $\theta \in \operatorname{Irr}\left(O_{p^{\prime}}(G)\right)$ be under $\varphi$, let $\delta \in I B r(T \mid \theta)$ with $\delta^{G}=\varphi$, where $T$ is the stabilizer of $\theta$ in $G$, and let $b \in B l(T)$ be the block of $\delta$. If $T=G$, by Lemma 2 and Lemma 3, we have that, in both cases, $G$ is $p$-nilpotent and so every block and every character are nilpotent. If $T<G$, by induction and Lemma 1 and Lemma 4 , we have that $\varphi$ is nilpotent if and only if $\delta$ is nilpotent if and only if $b$ is nilpotent if and only if $B$ is nilpotent.

4. $\pi$-Blocks. Brauer himself considered the idea of generalizing $p$-blocks to $\pi$-blocks, for a set of primes $\pi$. Later, Robinson and others introduced several definitions of $\pi$-blocks. We will follow the Isaacs-Slattery's approach which certainly coincides with Robinson's when the group is $\pi$-separable. We refer the reader to [12] and [13], for definition, notation and further comments on the subject.

TheOREM 6. Let $G$ be a $\pi$-separable group and let $\varphi \in I_{\pi}(G)$ be nilpotent. Let $B$ be the $\pi$-block of $\varphi$. Then

(a) $\varphi$ is the only modular character in $B$.

(b) If $\delta^{G}=\varphi$, where $\delta \in I_{\pi}(K)$ has $\pi$-degree and $K$ has a normal Hall $\pi$-subgroup, then the map $\psi \rightarrow \psi^{G}$ from $\operatorname{Irr}\left(K \mid \delta_{O_{\pi}(K)}\right) \rightarrow$ $\operatorname{Irr}(B)$ is a bijection.

(c) With the notation of (b), $\left(\delta_{O_{\pi}(K)}\right)^{G}=\Phi_{\varphi}$. Thus, if $H$ is a Hall $\pi$-subgroup of $G$ containing $O_{\pi}(K)$, then $\left(\delta_{O_{\pi}(K)}\right)^{H} \in \operatorname{Irr}(H)$ is a Fong character for $\varphi$.

Proof. (a) Let $\theta \in \operatorname{Irr}(O)$ under $\varphi$, where $O=O_{\pi}(G)$. Let $\delta \in$ $I_{\pi}(T \mid \theta)$ with $\delta^{G}=\varphi$, where $T$ is the stabilizer of $\theta$ in $G$, and let $b$ be the $\pi$-block of $\delta$. If $T=G$, by Lemma $3, G$ has a normal Hall $\pi$-subgroup. Also by (2.8) of [12], we know that the modular characters in $B$ are the $\pi$-characters over $\theta$. By (6.3) of [7], it follows 
that $\varphi$ is the only one. On the other hand, if $T<G$, by Lemma 3, induction and Theorem (2.10) of [12], the result follows.

(b) We argue by induction on $|G|$.

Since $\delta$ has $\pi$-degree, we have that $\alpha=\delta_{O_{\pi}(K)} \in \operatorname{Irr}\left(O_{\pi}(K)\right)$.

Let $V=O O_{\pi}(K)$. Since $|O K: V|$ is a $\pi^{\prime}$-number, we have that $\alpha^{V}=\left(\delta^{O K}\right)_{V} \in \operatorname{Irr}(V)$. Since $\alpha$ is $K$-invariant, by (4.3) of [7], it follows that the map $\psi \rightarrow \psi^{O K}$ is a bijection from $\operatorname{Irr}(K \mid \alpha) \rightarrow$ $\operatorname{Irr}\left(O K \mid \alpha^{V}\right)$. Now let $\theta \in \operatorname{Irr}(O)$ be under $\alpha^{V}$ and let $\epsilon \in I_{\pi}(T \cap$ $O K \mid \theta)$ be such that $\epsilon^{O K}=\delta^{O K}$, where $T$ is the stabilizer of $\theta$ in $G$. If $\mu=\epsilon^{T}$, observe that $\mu \in I_{\pi}(T \mid \theta)$ and $\mu^{G}=\varphi$. By Lemma 4 , notice that $\mu$ is nilpotent. If $T=G$, by Lemma 3, we have that $O$ is a Hall $\pi$-subgroup of $G$. Also, $\varphi_{O}=\theta$, which forces $O K=G$. In this case, $V=O, \alpha^{V}=\theta$ and we know that $\psi \rightarrow \psi^{G}$ is a bijection from $\operatorname{Irr}(K \mid \alpha) \rightarrow \operatorname{Irr}(G \mid \theta)$. Since $\operatorname{Irr}(B)=\operatorname{Irr}(G \mid \theta)$, by $(2.8)$ of $[\mathbf{1 2}]$, in this case, we are done. So we may assume that $T<G$ and by induction we have that the map $\psi \rightarrow \psi^{T}$ is a bijection from $\operatorname{Irr}\left(T \cap O K \mid \epsilon_{T \cap V}\right) \rightarrow \operatorname{Irr}(b)$. Since $\epsilon_{T \cap V}$ is $T \cap O K$-invariant and induces $\alpha^{V}$, by (4.3) of [7], it follows that the map $\psi \rightarrow \psi^{O K}$ is a bijection from $\operatorname{Irr}\left(T \cap O K \mid \epsilon_{T \cap V}\right) \rightarrow \operatorname{Irr}\left(O K \mid \alpha^{V}\right)$ (observe that $(T \cap O K) V=O K$, because they have coprime indices). By the above and Theorem (2.10) of [12], we have that the map $\psi \rightarrow \psi^{G}$ is a bijection from $\operatorname{Irr}\left(T \cap O K \mid \epsilon_{T \cap V}\right) \rightarrow \operatorname{Irr}(B)$ and therefore so it is the map $\psi \rightarrow \psi^{G}$ from $\operatorname{Irr}\left(O K \mid \alpha^{V}\right) \rightarrow \operatorname{Irr}(B)$. This proves (b).

(c) By Lemma (2.3) of [10], it suffices to show that $\left(\delta_{O_{\pi}(K)}\right)^{G}=$ $\Phi_{\varphi}$. If $\chi \in \operatorname{Irr}(B)$, by (b), we have that $\chi^{o}=(\chi(1) / \varphi(1)) \varphi$. Then,

$$
\begin{aligned}
\Phi_{\varphi} & =\sum_{\chi \in \operatorname{Irr}(B)}(\chi(1) / \varphi(1)) \chi=\sum_{\psi \in \operatorname{Irr}\left(K \mid \delta_{O_{\pi}(K)}\right)}(\psi(1) / \delta(1)) \psi^{G} \\
& =\left(\sum_{\psi \in \operatorname{Irr}\left(K \mid \delta_{O_{\pi}(K)}\right)}(\psi(1) / \delta(1)) \psi\right)^{G} \\
& =\left(\left(\delta_{O_{\pi}(K)}\right)^{K}\right)^{G}=\left(\delta_{O_{\pi}(K)}\right)^{G} .
\end{aligned}
$$

It is not difficult to show that all Fong characters associated with $\varphi$ arise this way.

We think it is worth to remark that if an irreducible character $\chi$ is induced from a $p$-nilpotent character the $p$-block of $\chi$ need not 
to be nilpotent. For instance, consider $\chi$ an irreducible character of degree 3 in the symmetric group on four letters and $p=2$. The block of $\chi$ is the principal block which is not nilpotent (because $G$ is not $p$-nilpotent). However, $\chi$ is induced from a Sylow 2-subgroup of $G$.

5. An example. We mentioned above that if a block $b^{G}$ is defined and nilpotent, then $b$ needs not to be nilpotent. More surprisingly, if a block nilpotent $b$ covers a block $e, e$ needs not to be nilpotent (this fact was communicated to the author by L. Puig, and we take this opportunity for thanking him). We give an easy

EXAMPLE 7. Let $D=\langle x, y\rangle$ be the dihedral group of order 8 , with $C=\langle x\rangle$ of order 4 and $x^{y}=x^{-1}$ and let $D$ act on $P=\langle z\rangle$ of order 3 by $z^{y}=z^{-1}$ and $C$ acting trivially. Let $G=P D$ be the semidirect product and put $p=3$. Let $\lambda \in \operatorname{Irr}(C)$ of order 4 and $\hat{\lambda}=\lambda \times 1_{P} \in \operatorname{Irr}(P \times C)$. Then $\chi=(\hat{\lambda})^{G} \in \operatorname{Irr}(G)$. Observe that, by (7.1) of [7], $\chi \in B_{2}(G)$ and thus, $\varphi=\chi^{o} \in \operatorname{IBr}(G)$. Observe that $\varphi$ is nilpotent. Let $J=P\langle y\rangle$ and let $H=J \times Z \triangleleft G$, where $Z=\left\langle x^{2}\right\rangle$. Then $\chi_{H}=\mu_{1}+\mu_{2}$, where $\mu_{1} \in \operatorname{Irr}(H / P)$, and $\mu_{i}$ is linear. Then, $\mu_{i}$, which is normal constituent of a nilpotent character $\varphi$, is not nilpotent (since $H$ is not $p$-nilpotent). This shows that, in general, nilpotent characters do not lie over nilpotent characters. Also, $\mu_{i}^{G}=\varphi$, and hence the nonnilpotent block of $\mu_{i}$ induces the block of $\varphi$.

\section{REFERENCES}

[1] J.L. Alperin, Local representation theory, Cambridge University Press, 1986.

[2] J.L. Alperin and M. Broué, Local methods in block theory, Ann. Math., 110 (1979), 143-157.

[3] H.I. Blau, On block induction, J. of Alg., 104 (1986), 195-202.

[4] M. Broué and L. Puig, A Frobenius theorem for blocks, Invent. Math., 56 (1980), 117-128.

[5] W. Feit, The representation Theory of Finite Groups, North Holland, 1982.

[6] I.M. Isaacs, Character Theory of Finite Groups, Academic Press, New York, San Francisco, 1976. Characters of $\pi$-separable groups, J. Algebra, 86 (1984), 98-128. 
[8] — Fong characters in $\pi$-separable groups, J. Algebra, 99 (1986), 89-107.

[9] I.M. Isaacs and G. Navarro, Weights and vertices for characters of $\pi$ separable groups, to appear in J. Algebra.

[10] G. Navarro, Fong characters and correspondences in $\pi$-separable groups, Can. J. Math., 43 (1991), 405-412.

[11] L. Puig, Local block theory in p-solvable groups, Proc. of Symp. in Pure Math., 37 (1980), 385-388.

[12] M.C. Slattery, Pi-blocks of pi-separable groups, I, J. Algebra, 102 (1986), 60-77.

[13] _ , Pi-blocks of pi-separable groups, II, J. Algebra, 124 (1989), 236269.

Received December 10, 1992. Research partially supported by DGICYT.PB 90-0414-C03-01.

UNIVERSITAT DE VALENCIA

46100 BURJASSOT, VALENCIA

SPAIN

E-mail address: gabriel@vm.ci.uv.es 



\title{
PACIFIC JOURNAL OF MATHEMATICS
}

Founded by E. F. Beckenbach (1906-1982) and F. Wolf (1904-1989)

\section{EDITORS}

Sun-Yung Alice Chang (Managing Editor)

University of California

Los Angeles, CA 90095-1555

pacific@math.ucla.edu

F. Michael Christ

University of California

Los Angeles, CA 90095-1555

christ@math.ucla.edu

\section{Thomas Enright}

University of California

San Diego, La Jolla, CA 92093

tenright@ucsd.edu

Nicholas Ercolani

University of Arizona

Tucson, AZ 85721

ercolani@math.arizona.edu
Robert Finn

Stanford University

Stanford, CA 94305

finn@gauss.stanford.edu

Vaughan F. R. Jones

University of California

Berkeley, CA 94720

vfr@math.berkeley.edu

Steven Kerckhoff

Stanford University

Stanford, CA 94305

spk@gauss.stanford.edu
Martin Scharlemann

University of California

Santa Barbara, CA 93106 mgscharl@math.ucsb.edu

Gang Tian

Courant Institute

New York University

New York, NY 10012-1100

tiang@taotao.cims.nyu.edu

V. S. Varadarajan

University of California

Los Angeles, CA 90095-1555

vsv@math.ucla.edu

\section{SUPPORTING INSTITUTIONS}

\section{CALIFORNIA INSTITUTE OF TECHNOLOGY NEW MEXICO STATE UNIVERSITY \\ OREGON STATE UNIVERSITY \\ STANFORD UNIVERSITY \\ UNIVERSITY OF ARIZONA \\ UNIVERSITY OF BRITISH COLUMBIA \\ UNIVERSITY OF CALIFORNIA \\ UNIVERSITY OF HAWAII}

\author{
UNIVERSITY OF MONTANA \\ UNIVERSITY OF NEVADA, RENO \\ UNIVERSITY OF OREGON \\ UNIVERSITY OF SOUTHERN CALIFORNIA \\ UNIVERSITY OF UTAH \\ UNIVERSITY OF WASHINGTON \\ WASHINGTON STATE UNIVERSITY
}

The supporting Institutions listed above contribute to the cost of publication of this Journal, but they are not owners or publishers and have no responsibility for its contents or policies.

Manuscripts must be prepared in accordance with the instructions provided on the inside back cover.

The Pacific Journal of Mathematics (ISSN 0030-8730) is published monthly except for July and August. Regular subscription rate: $\$ 215.00$ a year (10 issues). Special rate: $\$ 108.00$ a year to individual members of supporting institutions.

Subscriptions, orders for back issues published within the last three years, and changes of subscribers address should be sent to Pacific Journal of Mathematics, P.O. Box 4163, Berkeley, CA 94704-0163, U.S.A. Prior back issues are obtainable from Kraus Periodicals Co., Route 100, Millwood, NY 10546.

The Pacific Journal of Mathematics at the University of California, c/o Department of Mathematics, 981 Evans Hall Berkeley, CA 94720 (ISSN 0030-8730) is published monthly except for July and August. Second-class postage paid at Berkeley, CA 94704, and additional mailing offices. POSTMASTER: send address changes to Pacific Journal of Mathematics, P.O. Box 6143, Berkeley, CA 94704-0163.

\author{
PUBLISHED BY PACIFIC JOURNAL OF MATHEMATICS at University of California, \\ Berkeley, CA 94720, A NON-PROFIT CORPORATION \\ This publication was typeset using AMS-LATEX, \\ the American Mathematical Society's TEX macro system. \\ Copyright (C) 1995 by Pacific Journal of Mathematics
}




\section{PACIFIC JOURNAL OF MATHEMATICS}

Volume $169 \quad$ No. $2 \quad$ June 1995

On Banach spaces $Y$ for which $B(C(\Omega), Y)=K(C(\Omega), Y)$

201

SHAMIM ISMAIL ANSARI

Convergence of infinite exponentials

219

GENNADY BACHMAN

Cohomologie d'intersection modérée. Un théorème de de Rham

235

Bohumil Cenkl, Gilbert Hector and Martintxo

SARALEGI-ARANGUREN

Kleinian groups with an invariant Jordan curve: $J$-groups

291

Ruben A. Hidalgo

Multiplicative functions on free groups and irreducible representations 311

M. GABRiella KuHN and Tim STEGer

A Diophantine equation concerning finite groups

MAOHUA LE

Nilpotent characters

343

GABRIEL NAVARRO

Smooth extensions and quantized Fréchet algebras

XiaOlU WANG 